\title{
COMPARISON OF SOIL CONTAMINATION AT THE SELECTED EUROPEAN COPPER MINES
}

\author{
Peter ANDRÁŠ ${ }^{1}$, Pavol MIDULA ${ }^{1}$, João X. MATOS ${ }^{3}$, Giuseppe BUCCHERI ${ }^{2}$, Marek \\ DRÍMAL ${ }^{1}$, Vojtech DIRNER ${ }^{1}$, Zuzana MELICHOVÁ ${ }^{1}$ \& Ingrid TURISOVÁ ${ }^{1}$ \\ ${ }^{1}$ Matej Bel University, Faculty of Natural Sciences, Banská Bystrica, Tajovského 40, Banská Bystrica, Slovakia; \\ peter.andras@umb.sk; marek.drimal@umb.sk; pavol.midula@umb.sk; vojtech.dirner@umb.sk \\ ${ }^{2}$ INAIL - Italian Workers Compensation Authority, UOT Napoli, Via Nuova Poggioreale - 80143 Napoli, Italy; g.buccheri@inail.it \\ ${ }^{3}$ Laboratório Nacional de Energia e Geologia (Portuguese Geological Survey), Ap. 14 7601-909 Aljustrel, Portugal, \\ joao.matos@ineg.pt
}

\begin{abstract}
This article reports the results of a study concerning contamination of the dump-fields by potentially toxic elements at five abandoned copper mines: Lubietová, Špania Dolina (Slovakia), Libiola, Caporciano (Italy) and São Domingos (Portugal). This paper offers an updated description of soil contamination at the individual deposits and indicates a possible solution of the derived environmental problems. Contamination of technosoils by PTEs at the dump-fields shows an irregular spatial distribution of Fe, Cr, Mn, Co, Ni, Cu, Zn, As, Cd, Sb and $\mathrm{Pb}$. Contents of PTEs often exceed both national and EU law limits. Whereas at Lubietová, Špania Dolina and Caporciano the environmental risk is limited, at Libiola and São Domingos it seems to be very heavy. The technosoil (slag) of the dump-fields is not well aerated and the soil colloids have (except for Špania Dolina) negative surface charge, so they are suitable for PTEs sorption. The main environmental risk in the mining area of Sao Domingos district is the long time formation of acid mine drainage water and the high $\mathrm{Zn}$, As and $\mathrm{Pb}$ contents. The release of PTEs to the environmental components may be limited by phytostabilization and immobilization of metals, by suitable admixtures into the technosoil/slag. The environmental situation at Caporciano is not so compromised as at São Domingos mining area. Phytostabilization will be able to stop erosion, and installation of wetlands under the dump will stop the release of $\mathrm{Cu}$ to the environment.
\end{abstract}

Key words: abandoned $\mathrm{Cu}$ mines; potentially toxic elements; dump-fields; technosoil; environmental risk

\section{INTRODUCTION}

Slovakia, Italy and Portugal are known in Europe for their mining tradition. In all three countries, in fact, heavy environmental problems have derived from mining, treatment and processing activities. The presented article is focused on comparison of the poten-tially toxic elements (PTEs) contamination of the dump-fields at the selected five abandoned Cu-deposits (Lubietová, Špania Dolina, Caporciano, Libiola, and São Domingos; (Fig. 1), with respect to their environmental risk.

Lubietová is located in the central part of the Western Carpathians. It was one of the most important copper mining districts in the past. The volcanosedimentary Cu-(Ag-) mineralization is genetically connected with the basic, intermediate and acid Permian volcanism. There, metals were mobilized by granite intrusion during the Alpine orogeny. The main ore minerals are chalcopyrite, tetrahedrite (often Ag-bearing) and pyrite (Andráš et al., 2008; 2009). The mine was closed in 1915. The dump-field material on the steep slope consists of mixture of strongly weathered greywacke fragments with coarse-grained sandy gravel, which contain rests of the ore minerals.

The landscape relief at Špania Dolina is significant-ly changed by mining activity since Bronze age up to the $20^{\text {th }}$ century. The extractive activities affected a total area of $4 \times 1.5 \mathrm{~km}^{2}$. The most important pyrite mineralization of Triassic age is rich with chalcopyrite, tetrahedrite, ankerite, Cu-sulphides and $\mathrm{Cu}$-carbonates, as malachite and azurite. These minerals are still present in the dump-material. Among the large heap-fields, the most important one from the viewpoint of the environmental risk is the Maximilian heap, immediately above Špania Dolina village. It consists of the coarse-grained rock-fragments and sandy gravel (Nagyová et al., 2013). The exploitation of ore in Špania Dolina finished in 1888. 

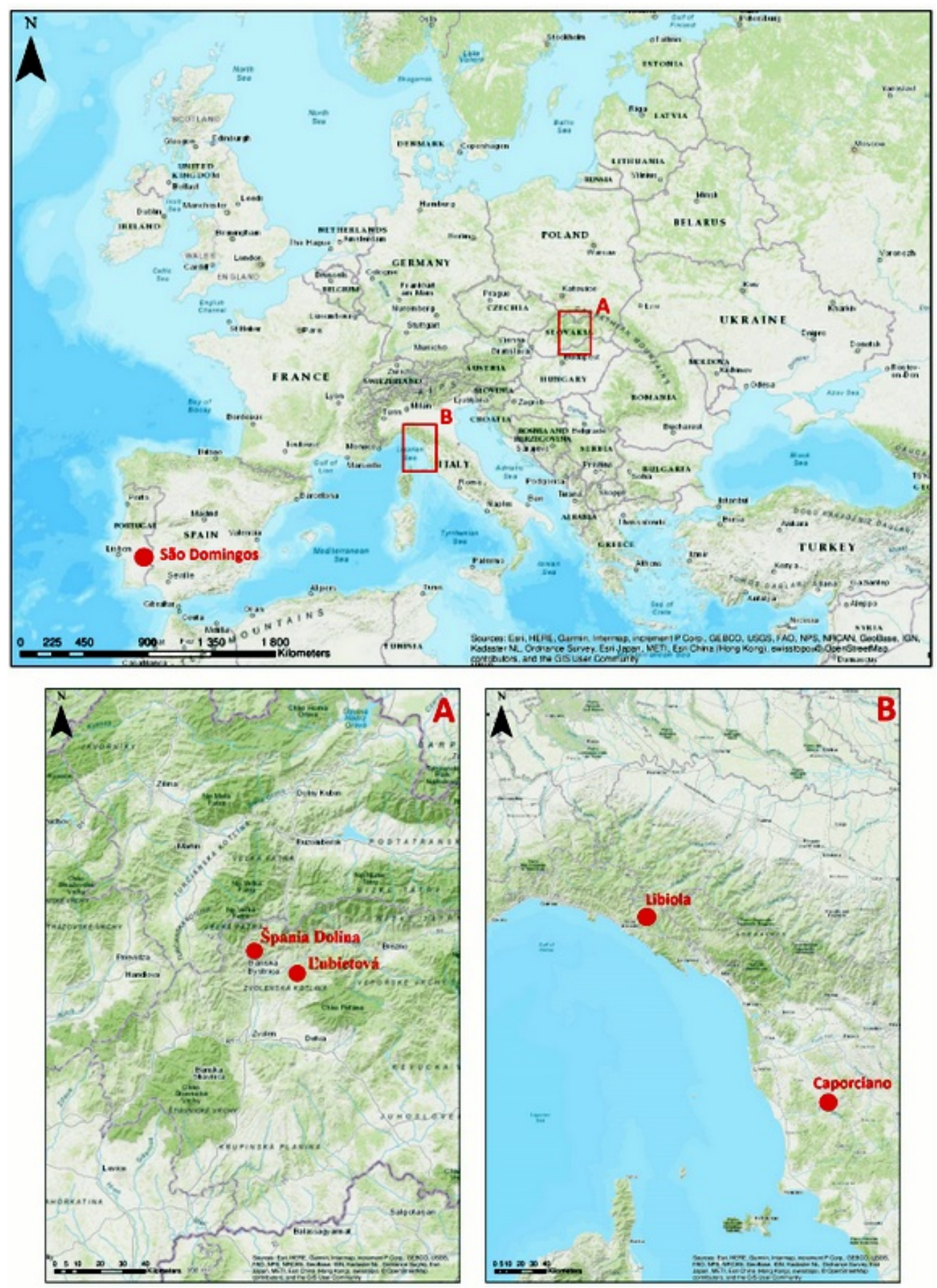

Figure 1. Localization of the studied abandoned Cu-deposits (Processed in ArcGIS 10.5)

Libiola deposit may be classified as a stratabound volcanic-associated massive sulphide deposit (VMS), where sulphide mineralization is mainly associated to pillow basalts and basaltic breccias and, subordinately, to serpentinitic rocks of ophiolites of Internal Ligurian Units (Ferrario et al., 1980). The deposit was exploited both by the system of numerous galleries and in the open-pit mine on the top of the mountain range. The stratified dump-field material, covering an area of $0.5 \mathrm{~km}^{2}$, is heterogeneous in its grain size and lithology of the rocks (mixture of ultramafic and Fe-rich phases; Dinelli et al., 2001). The dumped material contains significant amounts of chalcopyrite and pyrite ore waste (Marescotti et al., 2010). The end of mining activity at Libiola is dated to 1961 (Galli \& Penco, 1966).

Caporciano deposit, near Montecatini Val di Cecina, was the most important $\mathrm{Cu}$ deposit in Europe 
in the $19^{\text {th }}$ century (Riparbelli, 1980). In 1963, the mine was definitively closed. Caporciano mine may be considered as a remobilization deposit, showing features of medium temperature hydrothermal origin, whose mining importance is connected to the extraction of three sulphides: chalcopyrite, bornite and chalcocite (De Michele et al., 1987). The deposit was divided into two different mines: the large open-pit mine and underground galleries whose total longitude exceeded $30 \mathrm{~km}$ (Klemm et al., 1982). The spoil-heap consists of grey sandy material and, because of the steep slope of its surface and limited plant cover, it is strongly affected by erosion (Buccheri et al., 2014a).

São Domingos belongs to the most important $\mathrm{Cu}$-mineralizations of a metallogenetic province in the Iberian Pyrite Belt (Sáez et al., 1999; Álvarez-Valero et al., 2008). It was exploited since Roman Age. As the demand for copper grew in the $19^{\text {th }}$ century, the exploitation was renewed after the assignation of the mining concession to the English company Mason \& Barry in 1859. The massive pyrite-chalcopyrite ore body forms the top parts of the Volcano-Sedimentary Complex, consisting of felsic, intermediate to basic volcanic rocks, and black shales (Matos et al., 2006a, 2008). The main economically important ores were: pyrite, sphalerite, chalco-pyrite, galena, arsenopyrite and sulfosalts (Matos et al., 2006b; Webb, 1958). Spacious areas without any or with poor vegetation cover reflect the strong negative impact of the longterm mining activities. The slag and technosoil contain high PTEs content (Álvarez-Valero et al., 2008; Mateus, et al. 2011; Carvalho, 1971). All the country is markedly affected by acid mine drainage water (Abreu et al., 2010). The great open-pit mine was closed in 1966 due to mineral depletion (Matos et al., 2006a).

For our study, we selected a set of PTEs which are typical for all five studied deposits: $\mathrm{Fe}, \mathrm{Cr}, \mathrm{Mn}$, Co, Ni, Cu, Zn, As, Cd, Sb and Pb.

\section{MATERIAL AND METHODS}

Sampling procedure involves sample collection and sample reduction. The soil material was collected according to a regular 20x20 m network in such a way that each final representative sample consists of 5 original samples, which were mixed together and subsequently homogenized. 33 samples from the dump-fields and 3 samples from the reference area were taken at Lubietová, and 40 dump-samples were collected at Špania Dolina. For the research, we also collected further 36 and 12 dump samples, as well as 3 and 2 samples from reference areas at Libiola and Caporciano. The set of samples was completed by 30 samples from São Domingos mining area and by 5 samples from its reference area. The samples were then dried at laboratory temperature.

The rinse $\mathrm{pH}$ and Eh were measured according to Sobek et al., (1978) in water. The paste $\mathrm{pH}$ and Eh were measured in $1 \mathrm{M} \mathrm{KCl}$ lixivium, using $\mathrm{pH}$-metre EUTECH Instruments. The determined $\mathrm{pH}$ and Eh values were calculated for standard hydrogen electrode.

For comparison of Eh values at different $\mathrm{pH}$, the $\mathrm{rH}_{2}$ factor was used, defined by Richter et al. (2003) as it follows: $\mathrm{rH}_{2}=\mathrm{Eh} / 30+2 \mathrm{pH}$. Well aerated soils are characterized by $\mathrm{rH}_{2}$ factor ranging between 28 and 34, whereas not aerated soils, are characterized by $\mathrm{rH}_{2}$ factor $<20$.

The $\mathrm{D}_{\mathrm{pH}}$ factor is defined as $\mathrm{pH}_{\mathrm{H} 2 \mathrm{O}}-\mathrm{pH}_{\mathrm{KCl}}$ difference (McNeill, 1992). Positive values of the $D_{\mathrm{pH}}$ factor indicate presence of soil colloids with negative surface charge and, vice versa, negative values of the $\mathrm{D}_{\mathrm{pH}}$ factor reflect colloids with positive charge.

The technosoil samples were homogenized and dried at laboratory temperature and consequently analysed for $\mathrm{Fe}, \mathrm{Cr}, \mathrm{Mn}, \mathrm{Co}, \mathrm{Ni}, \mathrm{Cu}, \mathrm{Zn}, \mathrm{As}, \mathrm{Cd}, \mathrm{Sb}$ and $\mathrm{Pb}$, using ICP-MS method, in ACME Laboratory (Vancouver, Canada).

$2 \mathrm{~g}$ of technosoil powder was wetted with a distilled $\mathrm{H}_{2} \mathrm{O}$ and subsequently digested to dry vapour in $\mathrm{H}_{2} \mathrm{O}-\mathrm{HF}-\mathrm{HClO}_{4}-\mathrm{HNO}_{3}$ mixture. $10 \mathrm{ml}$ of $50 \% \mathrm{HCl}$ was added to the samples and the solutions were heated on water bath. The well homogenized and limpid solution was refilled by $\mathrm{HCl}$ to exact volume and analysed by ICPMS in ACME laboratories (Vancouver, Canada).

\section{RESULTS}

At all investigated deposits, the results demonstrate high concentrations, except for $\mathrm{Fe}$, mainly of $\mathrm{Cr}, \mathrm{Mn}, \mathrm{Ni}, \mathrm{Cu}, \mathrm{Zn}, \mathrm{As}, \mathrm{Sb}$ and $\mathrm{Pb}$ on the dump-fields (Table 1, Fig. 2). The most contaminated technosoil is at São Domingos mining area, where the average concentrations of $\mathrm{Zn}, \mathrm{As}$ and $\mathrm{Pb}$ are, respectively, $960 \mathrm{mg} \cdot \mathrm{kg}^{-1}, 1191 \mathrm{mg} \cdot \mathrm{kg}^{-1}$ and 3122 $\mathrm{mg} \cdot \mathrm{kg}^{-1}$ (Tables $1 ; 2$ ).

The dump-field contamination is also very high at Libiola. The average PTEs contents are $16.54 \%$ as for $\mathrm{Fe}, 633 \mathrm{mg} \cdot \mathrm{kg}^{-1}$ as for $\mathrm{Cr}, 91 \mathrm{mg} \cdot \mathrm{kg}^{-1}$ as for Co and 451 $\mathrm{mg} \cdot \mathrm{kg}^{-1}$ as for Ni (Tables $1 ; 3$ ), whereas the highest Sb contents (on average $366.2 \mathrm{mg} \cdot \mathrm{kg}^{-1}$ ) were described at Špania Dolina deposit, where the most important source of Sb is tetrahedrite (Tables 1; 4).

Caporciano dump-field is firstly characterized by the most high $\mathrm{Cu}$ contents (on average $7302 \mathrm{mg} \cdot \mathrm{kg}^{-1}$ ), as well as by high $\mathrm{Mn}$ and $\mathrm{Cd}$ contents (on average 827 $\mathrm{mg} \cdot \mathrm{kg}^{-1}$ and $3.4 \mathrm{mg} \cdot \mathrm{kg}^{-1}$, respectively; Tables $1 ; 5$ ).

The metal contents at the dump-fields substantially exceed the background PTEs contents at the reference areas (Tables $2-6$ ). 
Table 1. Average metal contents at the studied dump-fields

\begin{tabular}{|c|c|c|c|c|c|c|c|c|c|c|c|}
\hline \multirow{2}{*}{ Deposit } & Fe & $\mathrm{Cr}$ & Mn & Co & $\mathrm{Ni}$ & $\mathrm{Cu}$ & Zn & As & Cd & Sb & $\mathbf{P b}$ \\
\hline & $\%$ & \multicolumn{10}{|c|}{$\mathrm{mg} \cdot \mathrm{kg}^{-1}$} \\
\hline Lubietová & 2.16 & 17 & 365 & 28 & 31 & 3662 & 23 & 179 & 0.1 & 31.8 & 30 \\
\hline Špania Dolina & 1.56 & 16 & 235 & 22 & 23 & 1500 & 38 & 234 & 0.1 & 366.2 & 26 \\
\hline Libiola & 16.54 & 633 & 639 & 91 & 451 & 3543 & 364 & 20 & 1.5 & 4.8 & 68 \\
\hline Caporciano & 2.70 & 136 & 827 & 32 & 115 & 7302 & 765 & 2 & 3.4 & $<3.0$ & 20 \\
\hline São Domingos & 10.06 & 64 & 393 & 21 & 23 & 656 & 960 & 1191 & 0.9 & 144.1 & 3122 \\
\hline
\end{tabular}

Table 2. ICP-MS analyses of technogenic soil from São Domingos mining area.

\begin{tabular}{|c|c|c|c|c|c|c|c|c|c|c|c|}
\hline \multirow{2}{*}{ Sample } & $\mathbf{F e}$ & $\mathrm{Cr}$ & Mn & Co & $\mathbf{N i}$ & $\mathbf{C u}$ & Zn & As & Cd & Sb & $\mathbf{P b}$ \\
\hline & $\%$ & \multicolumn{10}{|c|}{$\mathrm{mg} \cdot \mathrm{kg}^{-1}$} \\
\hline \multicolumn{12}{|c|}{ dump-field area } \\
\hline S-1 & 11.80 & 69 & 292 & 25.9 & 25.4 & 370 & 414 & 1004 & 0.4 & 125 & 2737 \\
\hline S-2 & 7.45 & 89 & 722 & 21.7 & 49.1 & 27 & 69 & 100 & 0.6 & 20 & 726 \\
\hline S-3 & 6.70 & 94 & 565 & 11.4 & 32.8 & 265 & 153 & 551 & 1.1 & 279 & 6695 \\
\hline S-4 & 9.59 & 54 & 174 & 11.4 & 14.4 & 793 & 772 & 1742 & 2.6 & 667 & 13500 \\
\hline S-5 & 8.22 & 49 & 125 & 12.7 & 13.2 & 874 & 1470 & 1965 & 3.8 & 1095 & 12000 \\
\hline S-6 & 20.43 & 35 & 426 & 66.3 & 12.1 & 1481 & 8760 & 916 & 3.9 & 388 & 8499 \\
\hline S-7 & 4.68 & 95 & 1164 & 18.1 & 52.5 & 148 & 97 & 76 & 0.2 & 9 & 137 \\
\hline S-8 & 6.62 & 103 & 298 & 6.6 & 34.6 & 113 & 88 & 628 & 0.2 & 34 & 360 \\
\hline S-9 & 9.41 & 36 & 16 & 0.6 & 1.1 & 57 & 16 & 1751 & 0.1 & 18 & 3194 \\
\hline S-10 & 8.44 & 31 & 101 & 3.9 & 8.6 & 257 & 74 & 1540 & 0.3 & 57 & 2305 \\
\hline S-11 & 16.64 & 73 & 92 & 2.5 & 4.9 & 216 & 85 & 3906 & 0.3 & 225 & 9407 \\
\hline S-12 & 25.24 & 66 & 182 & 49.2 & 6.5 & 1665 & 4503 & 1768 & 1.6 & 197 & 6255 \\
\hline S-13 & 10.25 & 50 & 1386 & 98.9 & 23.0 & 6205 & 312 & 343 & 0.8 & 50 & 1501 \\
\hline S-14 & 6.25 & 43 & 1878 & 36.7 & 62.3 & 1284 & 1463 & 273 & 2.6 & 44 & 715 \\
\hline S-15 & 20.57 & 64 & 268 & 73.9 & 16.0 & 1208 & 4162 & 507 & 0.8 & 48.7 & 1516 \\
\hline S-16 & 9.30 & 67 & 130 & 10.9 & 15.5 & 347 & 648 & 1474 & 0.4 & 62.8 & 2995 \\
\hline S-17 & 21.37 & 69 & 547 & 23.7 & 75.4 & 729 & 574 & 922 & 1.0 & 74.5 & 2008 \\
\hline S-18 & 10.15 & 70 & 220 & 8.2 & 15.2 & 546 & 217 & 2631 & 0.8 & 102.9 & 6712 \\
\hline S-19 & 14.78 & 20 & 56 & 9.2 & 2.9 & 279 & 815 & 2405 & 0.4 & 112.1 & 4025 \\
\hline S-20 & 8.76 & 98 & 59 & 3.4 & 8.3 & 230 & 168 & 1647 & 0.1 & 57.4 & 573 \\
\hline S-21 & 7.82 & 65 & 150 & 7.9 & 8.4 & 234 & 169 & 1187 & 0.4 & 65.0 & 719 \\
\hline S-22 & 3.29 & 58 & 126 & 4.0 & 15.6 & 335 & 75 & 362 & 0.1 & 43.0 & 516 \\
\hline S-23 & 13.46 & 59 & 165 & 44.4 & 9.0 & 757 & 2533 & 439 & 0.5 & 156.4 & 2123 \\
\hline S-24 & 5.43 & 55 & 170 & 3.6 & 12.7 & 82 & 68 & 1865 & 0.2 & 63.4 & 293 \\
\hline S-25 & 5.88 & 81 & 858 & 18.9 & 45.7 & 190 & 386 & 247 & 1.3 & 14.0 & 322 \\
\hline S-26 & 5.47 & 84 & 463 & 16.6 & 43.4 & 227 & 245 & 65 & 0.3 & 6.5 & 80 \\
\hline S-27 & 6.73 & 52 & 112 & 3.5 & 9.3 & 149 & 102 & 4366 & 0.9 & 234.1 & 1295 \\
\hline S-28 & 4.59 & 65 & 553 & 13.7 & 31.2 & 162 & 159 & 86 & 0.2 & 8.5 & 162 \\
\hline S-29 & 7.33 & 57 & 139 & 3.6 & 9.4 & 263 & 102 & 780 & 0.0 & 48.9 & 1790 \\
\hline S-30 & 5.33 & 66 & 346 & 9.2 & 33.5 & 187 & 94 & 189 & 0.1 & 19.4 & 511 \\
\hline \multicolumn{12}{|c|}{ reference area } \\
\hline S-1R & 3.41 & 51 & 813 & 14.2 & 25.1 & 28.4 & 53 & 26 & 0.3 & 4.2 & 52 \\
\hline S-2R & 1.78 & 26 & 513 & 5.9 & 13.7 & 24.5 & 43 & 8 & 0.3 & 3.7 & 18 \\
\hline S-3R & 4.84 & 75 & 2001 & 25.1 & 44.9 & 39.2 & 137 & 68 & 0.6 & 2.9 & 67 \\
\hline S-4R & 5.23 & 75 & 1497 & 27.0 & 47.3 & 39.1 & 139 & 84 & 0.6 & 4.4 & 70 \\
\hline S-5R & 4.90 & 105 & 2403 & 27.2 & 55.2 & 48.3 & 128 & 17 & 0.8 & 4.0 & 48 \\
\hline
\end{tabular}


Table 3. ICP-MS analyses of technosoil from Libiola deposit

\begin{tabular}{|c|c|c|c|c|c|c|c|c|c|c|c|}
\hline \multirow{2}{*}{ Sample } & $\mathbf{F e}$ & $\mathrm{Cr}$ & Mn & Co & $\mathbf{N i}$ & $\mathrm{Cu}$ & Zn & As & Cd & Sb & $\mathbf{P b}$ \\
\hline & $\%$ & \multicolumn{10}{|c|}{$\mathbf{m g} \cdot \mathbf{k g}^{-1}$} \\
\hline \multicolumn{12}{|c|}{ dump-field area } \\
\hline LB-1 & 15.89 & 222 & 960 & 33 & 80 & 945 & 410 & 10 & 1.0 & 4.0 & 74 \\
\hline LB-2 & 14.22 & 702 & 600 & 61 & 509 & 2991 & 228 & 6 & 9.4 & 2.3 & 16 \\
\hline LB-3 & 11.89 & 1118 & 3380 & 461 & 1022 & 11000 & 606 & 7 & 2.2 & 3.1 & 48 \\
\hline LB-4 & 15.09 & 830 & 4610 & 539 & 907 & 12400 & 691 & 14 & 2.7 & 3.1 & 69 \\
\hline LB-5 & 9.28 & 1158 & 1380 & 195 & 1104 & 7345 & 436 & 11 & 1.3 & 2.8 & 30 \\
\hline LB-6 & 10.82 & 1374 & 1010 & 108 & 713 & 5638 & 435 & 8 & 3.6 & 4.8 & 33 \\
\hline LB-7 & 19.08 & 1145 & 750 & 81 & 667 & 3238 & 265 & 14 & 0.5 & 7.7 & 113 \\
\hline LB-8 & 7.75 & 961 & 1232 & 85 & 982 & 1794 & 374 & 5 & 0.9 & 2.2 & 44 \\
\hline LB-9 & 6.38 & 752 & 1.068 & 73 & 732 & 1624 & 328 & 5 & 0.8 & 1.9 & 47 \\
\hline LB-10 & 10.02 & 989 & 952 & 101 & 1115 & 1765 & 402 & 4 & 1.1 & 2.5 & 27 \\
\hline LB-11 & 15.50 & 444 & 683 & 87 & 574 & 11800 & 2851 & 3 & 5.3 & 1.8 & 14 \\
\hline LB-12 & 19.25 & 86 & 18 & 21 & 9 & 323 & 22 & 10 & 0.7 & 2.0 & 137 \\
\hline LB-13 & 41.00 & 117 & 40 & 9 & 18 & 3343 & 219 & 110 & 1.0 & 1.7 & 40 \\
\hline LB-14 & 42.00 & 1121 & 385 & 34 & 308 & 12050 & 354 & 266 & 0.3 & 84.0 & 476 \\
\hline LB-15 & 21.13 & 190 & 198 & 10 & 32 & 1383 & 113 & 12 & 0.8 & 2.2 & 38 \\
\hline LB-16 & 35.23 & 114 & 112 & 28 & 29 & 2870 & 85 & 11 & 0.4 & 1.9 & 18 \\
\hline LB-17 & 15.70 & 386 & 417 & 38 & 250 & 2328 & 225 & 8 & 1.3 & 2.6 & 67 \\
\hline LB-18 & 16.98 & 641 & 469 & 71 & 645 & 4652 & 248 & 4 & 1.1 & 1.8 & 19 \\
\hline LB-19 & 16.91 & 529 & 477 & 79 & 433 & 4868 & 214 & 3 & 0.6 & 2.0 & 25 \\
\hline LB-20 & 23.88 & 763 & 233 & 87 & 437 & 6680 & 242 & 3 & 0.3 & 1.6 & 25 \\
\hline LB-21 & 15.43 & 267 & 800 & 59 & 154 & 2287 & 168 & 6 & 0.2 & 2.0 & 14 \\
\hline LB-22 & 15.82 & 238 & 738 & 40 & 89 & 1535 & 175 & 10 & 0.4 & 1.8 & 365 \\
\hline LB-23 & 23.70 & 69 & 27 & 4 & 8 & 1193 & 45 & 60 & 0.4 & 2.6 & 54 \\
\hline LB-24 & 24.34 & 423 & 330 & 31 & 191 & 3737 & 150 & 36 & 0.9 & 2.5 & 196 \\
\hline LB-25 & 22.54 & 348 & 573 & 59 & 160 & 4961 & 241 & 10 & 0.9 & 2.9 & 44 \\
\hline LB-26 & 14.09 & 516 & 770 & 66 & 433 & 2950 & 442 & 8 & 1.4 & 2.7 & 122 \\
\hline LB-27 & 14.88 & 593 & 544 & 81 & 661 & 4266 & 234 & 4 & 1.4 & 2.2 & 25 \\
\hline LB-28 & 17.67 & 575 & 485 & 76 & 496 & 4762 & 286 & 5 & 2.1 & 1.9 & 34 \\
\hline LB-29 & 18.74 & 481 & 485 & 81 & 356 & 5990 & 308 & 6 & 2.5 & 2.5 & 23 \\
\hline LB-30 & 20.48 & 656 & 452 & 94 & 471 & 6880 & 286 & 5 & 2.3 & 1.3 & 32 \\
\hline LB-31 & 11.80 & 1225 & 1202 & 114 & 717 & 1884 & 345 & 5 & 1.5 & 1.0 & 19 \\
\hline LB-32 & 14.21 & 245 & 846 & 35 & 127 & 2291 & 247 & 5 & 0.6 & 2.7 & 21 \\
\hline LB-33 & 15.93 & 303 & 1177 & 153 & 224 & 5347 & 579 & 8 & 1.8 & 2.2 & 16 \\
\hline LB-34 & 14.00 & 559 & 614 & 83 & 619 & 4108 & 227 & 5 & 0.6 & 2.2 & 26 \\
\hline LB-35 & 18.83 & 376 & 354 & 42 & 231 & 3065 & 195 & 10 & 0.6 & 2.0 & 29 \\
\hline LB-36 & 14.80 & 734 & 481 & 59 & 724 & 6337 & 420 & 11 & 3.0 & 2.0 & 72 \\
\hline \multicolumn{12}{|c|}{ reference area } \\
\hline LB-1R & 9.86 & 111 & 627 & 132 & 483 & 18 & 7 & 6 & 1.1 & 0.1 & 17 \\
\hline LB-2R & 12.97 & 269 & 509 & 68 & 284 & 2543 & 148 & 3 & 0.4 & 2.0 & 15 \\
\hline LB-3R & 5.15 & 455 & 603 & 38 & 435 & 448 & 189 & 6 & 0.3 & 1.0 & 32 \\
\hline
\end{tabular}

The soil reaction is mainly characterized by $\mathrm{pH}$ and Eh. The $\mathrm{pH}$ and Eh range and average values are shown in Table 7. The results show that the most acid conditions are at São Domingos (average $\mathrm{pH}_{(\mathrm{H} 2 \mathrm{O})}=$ 4.29; $\mathrm{pH}_{(\mathrm{KCl})}=3.95$ ) and L'ubietová (average $\mathrm{pH}_{(\mathrm{H} 2 \mathrm{O})}=$ 4.74; $\left.\mathrm{pH}_{(\mathrm{KCl})}=4.94\right)$ deposits. At Caporciano deposit. it was found that conditions are the closest to the neutral values (average $\mathrm{pH}_{(\mathrm{H} 2 \mathrm{O})}=6.56 ; \mathrm{pH}_{(\mathrm{KCl})}=5.99$ ).

The average redox potential $\mathrm{Eh}_{(\mathrm{H} 2 \mathrm{O})}$ ranges from 100.64 (at Lubietová). to 62.85 (at Špania
Dolina). to 67.71 (at Libiola) to 15.01 (Caporciano). up to 153.32 (at São Domingos). $\mathrm{Eh}_{(\mathrm{KCl})}$ ranges from 58.00 (at Caporciano) to 173.90 (at São Domingos). Both the $\mathrm{Eh}_{(\mathrm{KCL})}$ values and the $\mathrm{D}_{\mathrm{ph}}$ and $\mathrm{rH}_{2}$ factors are presented in Table 8.

\section{DISCUSSION}

Basic informative data about the contamination of the dump-fields by PTEs at selected deposits were 
Table 4. ICP-MS analyses of samples from Maximilian dump-field (Špania Dolina)

\begin{tabular}{|c|c|c|c|c|c|c|c|c|c|c|c|}
\hline \multirow{2}{*}{ Sample } & Fe & $\mathrm{Cr}$ & Mn & Co & $\mathrm{Ni}$ & $\mathrm{Cu}$ & Zn & As & Cd & Sb & $\mathbf{P b}$ \\
\hline & $\%$ & \multicolumn{10}{|c|}{$\mathbf{m g} \cdot \mathbf{k g}^{-1}$} \\
\hline \multicolumn{12}{|c|}{ dump-field area } \\
\hline MX-1 & 1.67 & 16 & 262 & 22.6 & 23.2 & 2713 & 51 & 347 & 0.2 & 533 & 43.9 \\
\hline MX-2 & 1.58 & 16 & 200 & 20.5 & 24.6 & 1541 & 31 & 277 & 0.1 & 451 & 20.8 \\
\hline MX-3 & 1.32 & 15 & 102 & 13.7 & 22.1 & 1241 & 27 & 174 & 0.1 & 311 & 15.4 \\
\hline MX-4 & 1.67 & 19 & 275 & 23.9 & 23.0 & 1065 & 32 & 204 & 0.2 & 330 & 25.3 \\
\hline MX-5 & 1.53 & 16 & 244 & 34.5 & 30.2 & 2057 & 46 & 272 & 0.2 & 417 & 15.3 \\
\hline MX-6 & 1.77 & 15 & 250 & 14.5 & 17.0 & 603 & 35 & 116 & 0.1 & 167 & 16.7 \\
\hline MX-7 & 1.81 & 15 & 313 & 15.2 & 17.8 & 741 & 31 & 230 & 0.1 & 410 & 23.4 \\
\hline MX-8 & 2.01 & 16 & 359 & 16.1 & 15.8 & 559 & 38 & 94 & 0.1 & 144 & 18.0 \\
\hline MX-9 & 1.80 & 15 & 351 & 18.2 & 18.0 & 981 & 32 & 130 & 0.1 & 208 & 16.6 \\
\hline MX-10 & 1.83 & 15 & 361 & 33.3 & 30.3 & 1859 & 51 & 229 & 0.2 & 367 & 18.8 \\
\hline MX-11 & 1.91 & 17 & 352 & 35.4 & 33.8 & 2377 & 55 & 297 & 0.2 & 477 & 17.2 \\
\hline MX-12 & 1.29 & 15 & 164 & 21.7 & 23.2 & 722 & 27 & 138 & 0.1 & 195 & 22.2 \\
\hline MX-13 & 1.31 & 17 & 146 & 13.1 & 19.2 & 855 & 32 & 124 & 0.1 & 402 & 27.2 \\
\hline MX-14 & 1.43 & 17 & 162 & 11.3 & 18.5 & 639 & 28 & 105 & 0.2 & 168 & 37.1 \\
\hline MX-15 & 1.49 & 17 & 223 & 22.0 & 21.6 & 716 & 34 & 195 & 0.1 & 259 & 32.2 \\
\hline MX-16 & 1.86 & 18 & 427 & 31.9 & 20.4 & 1035 & 44 & 235 & 0.2 & 340 & 34.7 \\
\hline MX-17 & 1.47 & 14 & 256 & 29.7 & 23.3 & 1157 & 38 & 192 & 0.1 & 287 & 21.9 \\
\hline MX-18 & 1.25 & 14 & 164 & 20.7 & 19.3 & 948 & 34 & 258 & 0.1 & 382 & 31.0 \\
\hline MX-19 & 1.63 & 13 & 336 & 30.4 & 24.8 & 2126 & 51 & 217 & 0.2 & 387 & 15.9 \\
\hline MX-20 & 1.75 & 20 & 355 & 23.3 & 18.8 & 1031 & 47 & 119 & 0.3 & 163 & 64.8 \\
\hline MX-21 & 1.73 & 20 & 331 & 21.1 & 20.9 & 823 & 38 & 115 & 0.1 & 175 & 51.8 \\
\hline MX-22 & 1.97 & 15 & 225 & 28.8 & 24.4 & 8502 & 99 & 983 & 0.4 & 1403 & 72.0 \\
\hline MX-23 & 1.29 & 13 & 62 & 9.6 & 27.4 & 1083 & 18 & 300 & 0.1 & 539 & 15.4 \\
\hline MX-24 & 1.73 & 20 & 194 & 27.1 & 31.6 & 2039 & 36 & 316 & 0.1 & 476 & 23.2 \\
\hline MX-25 & 1.71 & 19 & 174 & 18.2 & 27.6 & 1582 & 27 & 377 & 0.0 & 588 & 28.2 \\
\hline MX-26 & 1.15 & 10 & 156 & 15.5 & 13.9 & 1319 & 26 & 185 & 0.0 & 306 & 16.6 \\
\hline MX-27 & 1.36 & 13 & 108 & 11.6 & 23.6 & 926 & 24 & 239 & 0.0 & 443 & 18.3 \\
\hline MX-28 & 1.94 & 17 & 366 & 30.2 & 26.4 & 1840 & 43 & 270 & 0.2 & 441 & 17.8 \\
\hline MX-29 & 1.46 & 15 & 186 & 20.7 & 19.9 & 820 & 29 & 225 & 0.1 & 324 & 23.6 \\
\hline MX-30 & 1.44 & 18 & 218 & 26.3 & 22.4 & 989 & 33 & 160 & 0.1 & 229 & 29.7 \\
\hline MX-31 & 1.69 & 18 & 302 & 30.8 & 21.9 & 1030 & 36 & 201 & 0.0 & 288 & 26.4 \\
\hline MX-32 & 1.29 & 16 & 179 & 22.9 & 22.1 & 1068 & 52 & 229 & 0.2 & 298 & 24.3 \\
\hline MX-33 & 1.12 & 15 & 91 & 12.1 & 19.0 & 605 & 32 & 168 & 0.1 & 229 & 22.0 \\
\hline MX-34 & 1.40 & 17 & 248 & 19.1 & 19.8 & 1064 & 38 & 213 & 0.2 & 266 & 21.2 \\
\hline MX-35 & 1.15 & 16 & 143 & 17.7 & 21.2 & 840 & 32 & 252 & 0.0 & 340 & 23.4 \\
\hline MX-36 & 1.19 & 17 & 169 & 28.7 & 26.2 & 2705 & 48 & 333 & 0.2 & 444 & 16.8 \\
\hline \multicolumn{12}{|c|}{ reference area } \\
\hline MX-1R & 1.16 & 8 & 112 & 4.9 & 10 & 96 & 22 & 44 & 0.0 & 111 & 8.8 \\
\hline MX-2R & 1.14 & 4 & 98 & 3.2 & 08 & 111 & 10 & 38 & 0.0 & 98 & 7.6 \\
\hline MX-3R & 1.11 & 6 & 122 & 5.5 & 09 & 87 & 9 & 18 & 00 & 88 & 10.1 \\
\hline
\end{tabular}


Table 5. ICP-MS analyses of technosoil from Caporciano deposit

\begin{tabular}{|c|c|c|c|c|c|c|c|c|c|c|c|}
\hline \multirow{2}{*}{ Sample } & $\mathbf{F e}$ & $\mathrm{Cr}$ & Mn & Co & $\mathrm{Ni}$ & $\mathrm{Cu}$ & $\mathrm{Zn}$ & As & Cd & Sb & $\mathbf{P b}$ \\
\hline & $\%$ & \multicolumn{10}{|c|}{$\mathbf{m g} \cdot \mathbf{k g}^{-1}$} \\
\hline \multicolumn{12}{|c|}{ dump-field area } \\
\hline MC-1 & 5.30 & 147 & 799 & 34 & 111 & 6388 & 766 & 2 & 3.1 & $<3$ & 12 \\
\hline MC-3 & 5.64 & 148 & 775 & 32 & 113 & 8290 & 697 & 3 & 3.2 & $<3$ & 27 \\
\hline MC-3 & 4.69 & 206 & 785 & 36 & 159 & 6663 & 540 & $<1$ & 2.4 & $<3$ & 12 \\
\hline MC-4 & 5.71 & 175 & 813 & 36 & 131 & 9337 & 947 & 2 & 4.0 & $<3$ & 27 \\
\hline MC-5 & 6.42 & 142 & 819 & 35 & 112 & 8619 & 797 & 3 & 3.0 & $<3$ & 23 \\
\hline MC-7 & 0.30 & 102 & 730 & 27 & 97 & 6379 & 718 & 1 & 3.7 & $<3$ & 13 \\
\hline MC-8 & 0.33 & 104 & 800 & 30 & 106 & 5137 & 675 & 1 & 2.7 & $<3$ & 27 \\
\hline MC-9 & 0.27 & 105 & 1250 & 32 & 145 & 5021 & 589 & 3 & 3.7 & $<3$ & 23 \\
\hline MC-10 & 0.33 & 132 & 820 & 30 & 97 & 8451 & 883 & 2 & 4.5 & $<3$ & 24 \\
\hline MC-11 & 0.38 & 112 & 890 & 33 & 107 & 11324 & 1064 & 3 & 4.1 & $<3$ & 12 \\
\hline MC-12 & 0.32 & 101 & 860 & 31 & 112 & 5985 & 784 & 2 & 2.5 & $<3$ & 25 \\
\hline MC-13 & 030 & 8 & 68 & 20 & 54 & 876 & 53 & 07 & 1.4 & $<3$ & 9 \\
\hline MC-14 & 034 & 7 & 102 & 18 & 44 & 549 & 166 & 12 & 28 & $<3$ & 11 \\
\hline
\end{tabular}

Table 6 (part 1) ICP-MS analyses of samples from Podlipa at L'ubietová

\begin{tabular}{|c|c|c|c|c|c|c|c|c|c|c|c|}
\hline \multirow{2}{*}{ Sample } & Fe & $\mathrm{Cr}$ & Mn & Co & $\mathbf{N i}$ & $\mathbf{C u}$ & Zn & As & Cd & Sb & $\mathbf{P b}$ \\
\hline & $\%$ & \multicolumn{10}{|c|}{$\mathbf{m g} \cdot \mathbf{k g}^{-1}$} \\
\hline \multicolumn{12}{|c|}{ dump-field area } \\
\hline P-1 & 2.01 & 12 & 264 & 27 & 29 & 2228 & 18 & 141 & 0.05 & 51.2 & 17.2 \\
\hline P-2 & 2.27 & 15 & 508 & 68 & 44 & 3784 & 19 & 140 & 0.05 & 53.1 & 16.1 \\
\hline P-3 & 1.73 & 13 & 207 & 18 & 28 & 1288 & 16 & 140 & 0.01 & 52.5 & 31.5 \\
\hline P-4 & 1.98 & 11 & 231 & 30 & 32 & 1757 & 11 & 163 & 0.07 & 37.6 & 22.6 \\
\hline P-5 & 2.36 & 15 & 173 & 29 & 42 & 2216 & 14 & 207 & 0.10 & 61.5 & 72.4 \\
\hline P-6 & 2.68 & 19 & 225 & 11 & 30 & 1179 & 16 & 176 & 0.10 & 55.6 & 29.4 \\
\hline P-7 & 2.81 & 20 & 108 & 11 & 37 & 1128 & 15 & 252 & 0.07 & 47.2 & 31.9 \\
\hline P-8 & 2.08 & 12 & 150 & 16 & 38 & 2136 & 15 & 290 & 0.08 & 32.4 & 22.8 \\
\hline P-9 & 1.72 & 15 & 378 & 32 & 28 & 4056 & 22 & 102 & 0.09 & 13.6 & 13.9 \\
\hline P-10 & 1.67 & 15 & 238 & 23 & 24 & 4927 & 17 & 162 & 0.08 & 20.9 & 25.3 \\
\hline P-11 & 1.81 & 13 & 357 & 44 & 36 & 6.740 & 16 & 234 & 0.07 & 20.0 & 16.7 \\
\hline P-12 & 2.20 & 13 & 203 & 30 & 32 & 9032 & 15 & 256 & 0.08 & 27.2 & 15.3 \\
\hline P-13 & 2.14 & 13 & 199 & 29 & 43 & 6190 & 15 & 361 & 0.06 & 31.9 & 16.5 \\
\hline P-14 & 2.62 & 18 & 372 & 44 & 43 & 10196 & 21 & 304 & 0.08 & 27.3 & 19.3 \\
\hline P-15 & 2.21 & 15 & 223 & 38 & 36 & 9278 & 18 & 269 & 0.08 & 23.1 & 14.7 \\
\hline P-16 & 2.23 & 18 & 34 & 8 & 23 & 2736 & 10 & 338 & 0.09 & 34.2 & 21.1 \\
\hline P-17 & 2.90 & 25 & 225 & 35 & 33 & 4113 & 15 & 300 & 0.08 & 42.5 & 29.8 \\
\hline P-18 & 2.60 & 22 & 245 & 30 & 32 & 4270 & 17 & 231 & 0.07 & 38.4 & 22.0 \\
\hline P-19 & 2.76 & 22 & 385 & 41 & 43 & 6346 & 18 & 261 & 0.10 & 31.4 & 24.7 \\
\hline P-20 & 2.77 & 25 & 671 & 37 & 35 & 4584 & 26 & 160 & 0.10 & 29.6 & 26.8 \\
\hline P-21 & 2.28 & 24 & 474 & 19 & 31 & 3580 & 17 & 170 & 0.10 & 44.1 & 24.1 \\
\hline P-22 & 2.69 & 22 & 534 & 36 & 33 & 6216 & 25 & 205 & 0.10 & 35.5 & 30.3 \\
\hline
\end{tabular}


Table 6 (part 2) ICP-MS analyses of samples from Podlipa at L'ubietová

\begin{tabular}{|c|c|c|c|c|c|c|c|c|c|c|c|}
\hline P-23 & 2.29 & 31 & 366 & 19 & 21 & 2312 & 20 & 105 & 0.08 & 20.0 & 24.7 \\
\hline P-24 & 2.26 & 29 & 381 & 23 & 23 & 2402 & 20 & 121 & 0.09 & 22.1 & 28.2 \\
\hline P-25 & 1.76 & 17 & 768 & 24 & 33 & 1754 & 47 & 175 & 0.40 & 44.4 & 75.7 \\
\hline P-26 & 2.37 & 24 & 1500 & 44 & 31 & 1729 & 90 & 41 & 0.31 & 27.4 & 76.8 \\
\hline P-27 & 2.29 & 19 & 981 & 46 & 44 & 3648 & 121 & 182 & 0.60 & 38.1 & 105.3 \\
\hline P-28 & 1.76 & 18 & 198 & 14 & 23 & 2523 & 17 & 118 & 0.08 & 26.2 & 55.4 \\
\hline P-29 & 1.76 & 10 & 282 & 16 & 18 & 2258 & 15 & 84 & 0.09 & 17.8 & 10.1 \\
\hline P-30 & 1.56 & 11 & 268 & 15 & 18 & 1949 & 13 & 70 & 0.08 & 16.4 & 9.0 \\
\hline P-31 & 1.60 & 13 & 280 & 20 & 19 & 1477 & 15 & 70 & 0.07 & 17.8 & 15.5 \\
\hline P-32 & 1.66 & 11 & 326 & 25 & 16 & 1163 & 18 & 38 & 0.08 & 16.7 & 20.4 \\
\hline P-33 & 1.41 & 11 & 301 & 15 & 16 & 1645 & 13 & 57 & 0.06 & 14.3 & 9.1 \\
\hline \multicolumn{12}{|c|}{ reference area } \\
\hline P-1R & 122 & 13 & 47 & 2 & 6 & 29 & 20 & 11 & 0.10 & 13.4 & 208 \\
\hline P-2R & 132 & 12 & 67 & 5 & 7 & 55 & 18 & 10 & 0.11 & 14.2 & 191 \\
\hline P-3R & 122 & 10 & 56 & 5 & 6 & 48 & 19 & 9 & 0.13 & 11.9 & 310 \\
\hline
\end{tabular}

Table 7. pH and Eh range and average values of technosoil from dump-fields

\begin{tabular}{|c|c|c|c|c|c|c|c|c|c|c|c|c|}
\hline \multirow{2}{*}{ Deposit } & \multicolumn{3}{|c|}{ pH(н2о) } & \multicolumn{3}{|c|}{$\mathrm{Eh}_{(\mathrm{H} 2 \mathrm{O})}(\mathrm{mV})$} & \multicolumn{3}{|c|}{$\mathbf{p H}_{(\mathrm{KCl})}$} & \multicolumn{3}{|c|}{$\mathrm{Eh}_{(\mathrm{KCl})}(\mathrm{mV})$} \\
\hline & & & $\overline{\mathrm{x}}$ & & & $\overline{\mathrm{X}}$ & & & $\overline{\mathrm{x}}$ & & & $\overline{\mathrm{X}}$ \\
\hline L'ubietová & 3.75 & 6.32 & 4.64 & -5 & 156 & 100.64 & 3.82 & 5.96 & 4.86 & -16 & 159 & 90.60 \\
\hline Š Dolina & 4.32 & 6.91 & 5.29 & -42 & 127 & 62.85 & 4.26 & 6.75 & 5.30 & -36 & 130 & 62.63 \\
\hline Libiola & 4.01 & 6.07 & 5.21 & 12 & 146 & 67.71 & 3.95 & 6.18 & 5.16 & 4 & 147 & 69.29 \\
\hline Caporciano & 5.99 & 7.13 & 6.56 & -12 & 42 & 15.01 & 4.93 & 7.00 & 5.99 & -4 & 111 & 58.00 \\
\hline S Domingos & 2.74 & 7.10 & 4.29 & -10.7 & 243.4 & 153.32 & 2.69 & 246.5 & 3.95 & -5.6 & 166.4 & 173.90 \\
\hline
\end{tabular}

Table $8 . \mathrm{D}_{\mathrm{ph}}$ and $\mathrm{rH}_{2}$ factors range and average values in technosoil from dump-fields

\begin{tabular}{|c|r|r|r|r|r|r|}
\hline \multirow{2}{*}{ Deposit } & \multicolumn{3}{|c|}{ D $_{\text {ph }}$} & \multicolumn{3}{|c|}{ rH $_{\mathbf{2}}$} \\
\cline { 2 - 7 } & \multicolumn{2}{|c|}{ values range } & \multicolumn{1}{|c|}{$\overline{\mathrm{x}}$} & \multicolumn{2}{|c|}{ values range } & \multicolumn{1}{c|}{$\overline{\mathrm{x}}$} \\
\hline Lubietová & -9.59 & 0.84 & 0.22 & 13.22 & 13.93 & 13.58 \\
\hline Špania Dolina & -0.42 & 0.45 & -0.01 & 9.75 & 12.83 & 12.33 \\
\hline Libiola & -0.27 & 0.31 & 0.04 & 12.47 & 12.80 & 12.63 \\
\hline Caporciano & 0.01 & 1.19 & 0.58 & 13.44 & 19.29 & 14.21 \\
\hline São Domingos & 0.05 & 0.60 & 0.32 & 11.66 & 14.72 & 13.61 \\
\hline
\end{tabular}

published by many authors. For example the first information about the PTEs content in technosoil at Lubietová and Špania Dolina was presented by Andráš et al., (2008; 2009) and Nagyová et al., (2013) Some information about waste-heaps at Libiola and Caporciano was presented by Marescotti et al (2010) and Buccheri et al., (2014a). Most available information was published about São Domingos deposit by Matos et al., (2006a) According to Mateus et al., (2011) the reddish clay-rich slag contains up to $30-40 \%$ Fe up to $17 \% \mathrm{Zn} 09 \% \mathrm{~Pb}$ and $05 \% \mathrm{Cu}$. The present research (Table 2) confirmed these data. The extraordinarily high $\mathrm{Zn}$ As and $\mathrm{Pb}$ contents at the dump-field of São Domingos mining area are caused by the presence of the main ore minerals in the technosoil: pyrite sphalerite chalcopyrite galena arsenopyrite and different sulfosalts (Matos et al. 2006b).

The sandy-gravelly sediments at Libiola contain high amounts of chalcopyrite- and pyrite-rich material. The mixture of secondary Fe-oxydes and Fe-oxyhydroxides influences the high $\mathrm{Fe}$ content whereas the main source of $\mathrm{Cr} \mathrm{Co}$ and $\mathrm{Ni}$ is pyrite (Marescotti et al., 2010). The source of the highest Sb content described at Špania Dolina deposit is tetrahedrite. 

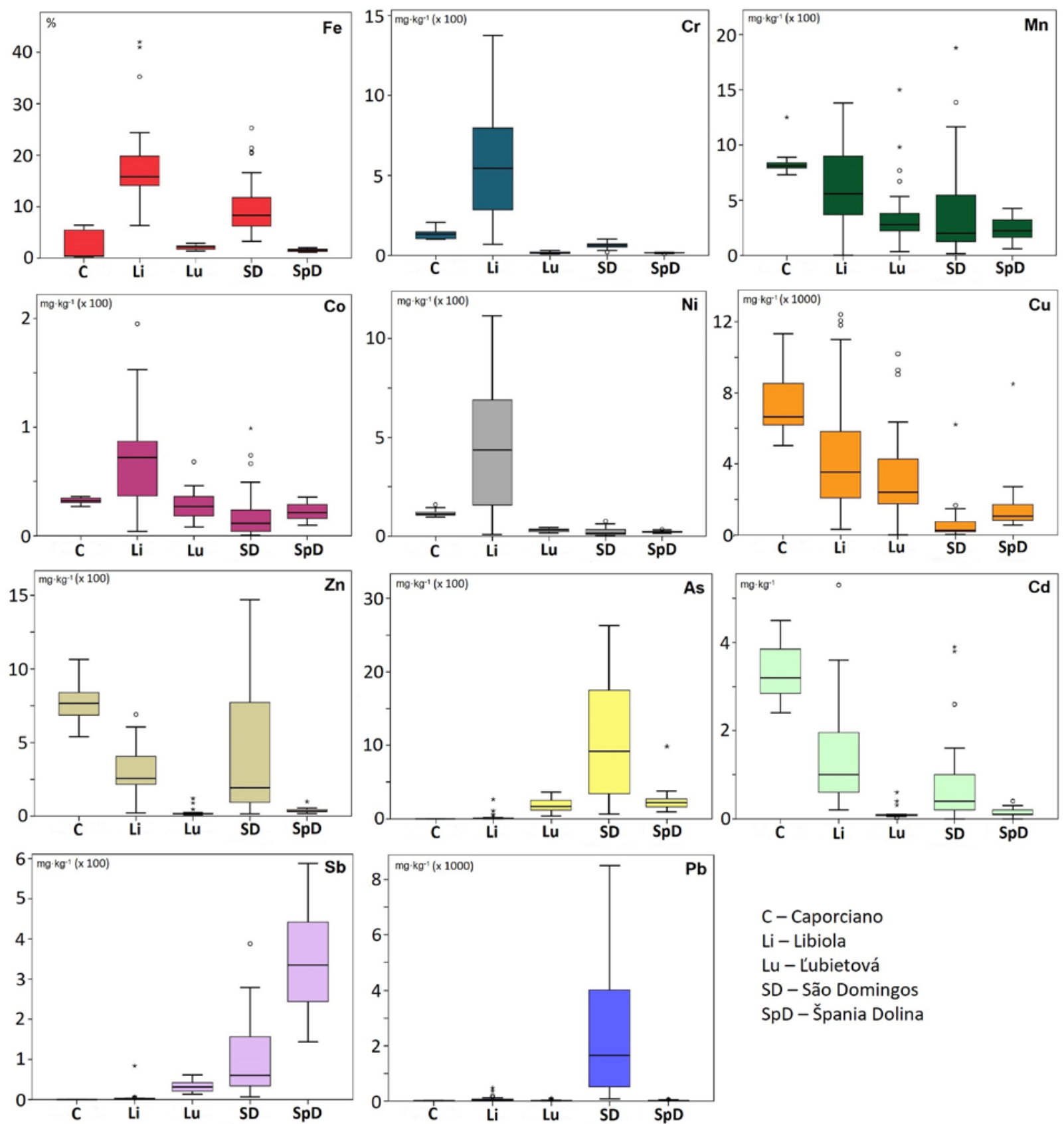

$$
\begin{aligned}
& \text { C-Caporciano } \\
& \text { Li-Libiola } \\
& \text { Lu - Ĺubietová } \\
& \text { SD - São Domingos } \\
& \text { SpD - Špania Dolina }
\end{aligned}
$$

Figure 2. Box-and-whisker plots, showing the difference of PTEs distribution among the investigated study areas. The outlayers describing the extremest values were excluded in order to improve the transparency of graphic outputs.

Caporciano dump-field is firstly characterized by the highest $\mathrm{Cu} \mathrm{Mn}$ and $\mathrm{Cd}$ contents (on average 7302 $\mathrm{mg} \cdot \mathrm{kg}^{-1} 827 \mathrm{mg} \cdot \mathrm{kg}^{-1}$ and $3.4 \mathrm{mg} \cdot \mathrm{kg}^{-1}$ respectively).

The red and brown colours at São Domingos and Libiola dump-fields indicate oxic conditions but the technosoil is not sufficiently aerated and the range of the Eh values (-10 7 to 243 4; Table 7) is typical for anoxic soils (Alloway 1992). The $\mathrm{rH}_{2}$ factor $<20$ (Richter et al., 2003) also confirms the same conclusions. According to McNeill (1992) the mostly positive $\mathrm{D}_{\mathrm{pH}}$ values at all deposits except for Špania Dolina (Table
8) indicate presence of soil colloids with negative charge so they have suitable sorption ability for metal cations. Data about the free sorption capacity of clay minerals at Lubietová deposit was also published by Andráš et al., (2009).

Results of analyses show that contents of heavy metals are irregular and in most cases exceed national law limits as well as EU limits for soils according to the Council Directive 86/78/EEC.

Repeated sampling at L'ubietová and Špania Dolina revealed great chemical variations and 
irregularities particularly in the dump-field area depending on weathering process PTEs migration ability formation of secondary minerals and sorption capacity of some minerals. The waste shows some selfcleaning ability a great part of PTEs is fixed in porous material in Fe-hydroxides and in clay minerals still showing an important free sorption capacity. For this reason it is not effective to interfere with dump materials (Andráš et al., 2008; 2012; Nagyová et al., 2013). The main environmental risk at Lubietová is represented by contamination of groundwater by PTEs mainly by the very toxic forms $\mathrm{As}^{3+}$ and $\mathrm{Sb}^{3+}$ (Franková et al., 2012). In order to eliminate this risk a $\mathrm{Fe}^{0}$-barrier was installed in the valley under the dump-field (Kupka et al., 2015). The dam system was installed to prevent the erosion caused by heavy rain and inappropriate technical interventions in the early 90 s.

Similar conditions can be described for Špania Dolina. As presented by Franková et al., (2012), the main risk is caused by contamination of grounwater by $\mathrm{As}^{3+}$ and $\mathrm{Sb}^{3+}$ from the $\mathrm{Cu}$-ores. No effective measures have been performed until now.

At Libiola the significant contents of $\mathrm{Fe} \mathrm{Cu} \mathrm{Cr}$ $\mathrm{Co} \mathrm{Ni}$ as well as of $\mathrm{Zn}$ As and $\mathrm{Mn}$ in the technosoil from the dump-field are released during the weathering process into the acid mine drainage water which is mixed with the slightly alkaline surface water (Gromolo river) with pH 8 (Dinelli et al., 2001; Buccheri et al., 2014a) in the valley near the inhabited area. The $\mathrm{pH}$ change and the precipitation of the amorphous Fe-phases (e g ferrihydrite schwertmannite) enable sorption of PTEs on their surface so that water shows acceptable quality in the inhabited area. Remobilization of PTEs should not be expected (Dinelli et al., 2001). It should be important to stabilize the slope (under the open-pit mine) on which the waste material is situated either by technical means or by suitable vegetation cover in order to limit PTEs release to the country components.

The main environmental risk at Caporciano is due to the heavy erosion of the heap that is situated in a terrain depression. The several pioneer plant species (dwarf species of Pinus spp. and Quercus spp.) are not able to stabilize the dump-material (Buccheri et al., 2014b). The deposit is situated high on the mountains and the nearest populated area is relatively far (about $2 \mathrm{~km}$ ) thus the relatively mobile $\mathrm{Cu}$ influences only the vineyards under the dump-field slope. There is neither creek nor river in the surrounding area thus the environmental risk due to diffusion of PTEs is limited. On the other hand a little marsh under the dump is saturated by sewage water from the hotel above the dump-field which drains the waste material. Adaptation of this marsh to anaerobic and aerobic wetland system could substantially eliminate the Cu-release to the country.

The most important environmental risk at São Domingos is due to the massive formation of acid drainage water. The greatest part of this water is collected in the flooded mine open pit. The rest is drained to the distant stream by $>14 \mathrm{~km}$ long channels. One part of the water is evaporated and the less substantial part of it reaches the creek and is diluted in nearby lake. Small dams store a significant volume of acid water. The enormous extent of the mining area does not enable simple solutions of the contaminated country remediation. One of the possible procedures how to reduce the negative impact of PTEs on the country components is phytostabilization of metals in the contaminated area using autochthon and pioneer plant species (Abreu et al., 2012; Andráš et al., 2018). Currently several admixtures are tested which can immobilize PTEs in the waste material.

\section{CONCLUSIONS}

The distribution of PTEs content in the dump material (technosoil and slag) of the individual studied abandoned Cu-deposits is different and, within each deposit, irregular. The highest $\mathrm{Zn}$, As and $\mathrm{Pb}$ contents were described in São Domingos mining area, whereas the dump-field material from Libiola is rich with $\mathrm{Fe}, \mathrm{Cr}, \mathrm{Co}, \mathrm{Ni}$ and the dump-field material from Caporciano is rich with $\mathrm{Cu}, \mathrm{Mn}$ and $\mathrm{Cd}$. The highest Sb content was found in the waste material from Špania Dolina deposit. The dump-field at Lubietová is contaminated by PTEs less than the dump-fields at the remaining four deposits.

The common features of the technosoil from these dump-fields are their limited aeration and anoxic conditions. The soil colloids are characterized (except for Špania Dolina) by negative charge and have suitable sorption ability for metal (PTEs) cations. Most of the PTEs contents exceed the EU law limits for the soil.

At Lubietová and Špania Dolina deposits, the main risk is represented by the intoxication of the groundwater by As and Sb. Libiola and Caporciano deposits are situated far from the inhabited areas. At Libiola, the main risk is caused by formation of acid drainage water with high $\mathrm{Cr}, \mathrm{Co}, \mathrm{Ni}$ (and $\mathrm{Cu}$ ) content, but this water is consequently neutralised and the PTEs precipitate, so that the river in the closest villages has close to neutral $\mathrm{pH}$ values and contain acceptable amount of PTEs. At Caporciano, it would be suitable to stop the waste erosion by covering the dump-field with vegetation cover. The contamination of the slope under the dump by Cu could be limited by installation of a wetland system in 
the depression beneath the dump.

The most contaminated mining area is São Domingos. The main hazard for the environment is represented by the enormous amount of acid mine drainage water and by very high PTEs contents in the technosoil and slag, first of all by Zn, As and Pb. One of the possible remediation techniques, which could be able to limit the PTEs release to country components (soil, water, plants) is phytostabilization, by using pioneer plant species; another possible remediation technique is the application of various admixtures to immobilize PTEs in the technosoil and slag.

\section{Acknowledgement:}

The article was supported by grant No. 1/0291/19 of grant agency VEGA and by support of Individual Fellowship Call: H2020-MSCA-IF-2016.

\section{References}

Abreu, M., Batista, M. J., Magalhães, M. C. F. \& Matos, J. X. 2010. Acid Mine Drainage in the Portuguese Iberian Pyrite Belt. Cap. II In Mine Drainage and Related Problems Book, Editor Brock C. Robinson, Nova Science Publishers, ISBN: 978-1-60741-2854, New York, USA, 51p.

Abreu, M., Magalhaes, M. C., Santos, E. S. \& Batista, M. J. 2012. São Domingos Mine Wastes Phytostabilization Using Spontaneous Plant Species, $9^{\text {th }}$ International Symposium on Environmental Geochemistry. Aveiro, $15^{\text {th }}-21^{\text {th }}$ July 2012, $42-49$

Alloway, B. J. 1992. Heavy Metals in Soils. Environmental Pollution, Blackie Academic \& Professional, ISBN 978-94-007-4469-1, 368 p.

Álvarez-Valero, A.M., Pérez-López, R., Matos, J.X., Capitán, M.A., Nieto, J.M., Sáez, R., Delgado, J. \& Caraballo M. 2008. Potencial environmental impact at São Domingos mining district (Iberian Pyrite Belt, SW Iberian Peninsula): evidence from a chemical and mineralogical characterization. Environmen-tal Geology, 55, 1797-1809.

Andráš, P., Lichý, A., Križáni, I., Rusková, J., Ladomerský, J., Jeleň, S., Hroncová, E. \& Matúšková, L. 2008. Podlipa dump-field at Lubietová - land contamined by heavy metals (Slovakia). Carpathian Journal of Earth and Environmental Sciences, 3, 2, ISSN 1842-4090, 518.

Andráš, P., Lichý, A., Križáni, I. \& Rusková, J. 2009. The heavy metal sorption on clay minerals and risk of the AMD formation at the Reiner and Podlipa dumpfields at Lubietová deposit (Slovakia). Carpathian Journal of Earth and Environmental Sciences, 2009, 4, 2, ISSN-1842-4090, 133-147

Andráš P., Turisová I., Krnáč J., Dirner V., VolekováLalinská B., Buccheri G. \& Jeleň S. 2012. Hazards of heavy metal contamination at Lubieto-vá $\mathrm{Cu}$ deposit (Slovakia). Procedia Environmental
Sciences, 3-21.

Andráš, P., Matos, J. X., Turisová, I., Batista, M. J., Kanianska, R. \& Kharbish, S. 2018. The interaction of heavy metals and metalloids in the soilplant system in the São Domingos mining area (Iberian Pyrite belt, Portugal). Environmental Science and Pollution Research, https:/doi.org/10.1007/s11356-018-2205-x, 2061520630

Buccheri G., Andráš P. Jr., Andráš P., Dadová J. \& Kupka J. 2014a. Heavy metal contamination and its impact on plants at Caporciano Cu-mine (Montecatini Val di Cecina, Italy), Carpathian Journal of Earth and Environmental Sciences, November 2014, 9, 4, 73-81.

Buccheri, G., Andráš, P., Astolfi, M. L., Canepari, S., Ciucci, M. \& Marino, A. 2014b. Heavy metal contamination in water at Libiola abandoned copper mine, Italy. Romanian Journal of Mineral Deposits, 87, 1, ISSN 1220-5648, 65-70.

Carvalho, D. 1971. Mina de S. Domingos. Jazigos Minerais do Sul de Portugal, Livro-Guia nº4, 59-64.

De Michele, V. \& Ostroman, A. 1987. Mineral processing at Montecatini deposit from 1888 to 1938. Milano. Museo Civico Storia Naturale, 1-38.

Dinelli, E., Lucchini, F., Fabbri, M. \& Cortecci, G. 2001. Metal distribution and environmental problems related to sulfide oxidation in the Libiola copper mine area (Ligurian Apennines, Italy). Journal of Geochemical Exploration, 74, 1-3,141-152.

Ferrario A. \& Garuti G. 1980. Copper deposits in the basal breccias and volcano-sedimentary sequences of the Eastern Ligurian Ophiolites (Italy). Mineralium Deposita, 15, ISSN 1432-1866, 291303.

Franková, H., Čmielová, L., Klimko, T., Lacková, E. \& Andráš, P. 2012. Comparative study of $\mathrm{Cu}$, As and $\mathrm{Sb}$ toxicity between dump-fields of anandoned $\mathrm{Cu}$ deposits Lubietová and Špania Dolina (Central Slovakia). Carpathian Journal of Earth and Environmental Sciences, 7, 4, ISSN 1842-4090, 7988.

Galli, M. \& Penco, A. M. 1966. Le miniere di rame e di manganese della Liguria orientale. Atti dell'Accademia Ligure di Scienze e Lettere, serie V, 53, 215-247.

Klemm, D. D. \& Wagner, J. 1982. Copper deposit in ophiolites of southern Tuscany. Ofioliti, 7, ISSN 0391-2612, 331-336.

Kupka, J., Andráš, P., Dadová, J., Andráš, P. Jr. \& Demeter, D. 2015. Testing of the permeable $\mathrm{Fe}^{0}$ barrier at dump-field Podlipa (Lubietová Cudeposit, Slovakia). $19^{\text {th }}$ Conference on Environment and Mineral Processing. Journal of the Polish Mineral Engineering Society, No. 2, ISSN 16404920, 189-194.

Marescotti, P., Bullet, E., Bullet, A., Servida, D., Carbone, C., Grieco, G., De, L., Bullet, C. \& Lucchetti, G. 2010. Mineralogical and geochemical spatial analyses of a waste-rock dump at the Libiola 
$\mathrm{Fe}-\mathrm{Cu}$ sulphide mine (Eastern Liguria, Italy). Environmental Earth Sciences, 61, DOI: 10.1007/s12665-009-0335-7, 187-199.

Mateus, A., Pinto, A., Alves, L. C., Matos, J. X., Figueiras, J. \& Neng, N. 2011. Roman and modern slag at S. Domingos mine (IPB, Portugal): compositional features and impli-cations for their long-term stability and poten-tial re-use. International Journal of Environ-ment and Waste Management Inderscience Publishers Ltd, Vol. 8, n1/2, 39 p.

Matos, J. X. \& Martins, L. P. 2006a. Reabilitação ambiental de áreas mineiras do sector português da Faixa Piritosa Ibérica: estado da arte e perspectivas futuras. Boletín Geológico y Minero, Espanha, 117, 2, 289-304.

Matos, J. X., Soares, S. \& Claudino, C. $2006 \mathrm{~b}$. Caracterização Geológica-geotécnica da corta da mina de S. Domingos, FPI. X Cong. Nac. Geotécnica, Soc. Port. Geotecnia, Un. Nova, V. 3, 741-752.

Matos, J. X., Martins, L. P., Oliveira, J. T., Pereira, Z., Batista, M. J. \& Quental, L., 2008. Rota da pirite no sector português da Faixa Piritosa Ibérica. desafios para um desenvolvimento sustentado do turismo geológico e mineiro. Projecto RUMYS. programa CYTED. In: Carrion P (ed). Livro Rutas Minerales en Iberoamérica. Sup. Politécnica del Litoral. Guayaquil. Equador. 136-155.
Nagyová, I., Melichová, Z., Komadelová, T., Boháč, P. \& Andráš, P. 2013. Environmental assessment of impacts by old copper mining activities - a case study at Špania Dolina Starohorské Mts., Slovakia, Carpathian Journal of Earth and Environmental Sciences, 8, 4, $101-108$

McNeill, J. D. 1992. Rapid. accuratemapping of soilsalinity by electromagneticground-conduc-tivity meters. In: Topp, G. C. et al. (eds): Advances in measurement of soil-physical properties: Bringingtheoryintopractice. SSSA Spec. Publ. 30. SSSA. Madison WI, 209-229.

Richter, R. \& Hlůšek, J. 2003. Soil floor. Ústav zemědelských a potravinářských informací, Ministerstvo zemědelství, Praha, 36 p. (in Czech)

Riparbelli A. 1980. Storia di Montecatini Val di Cecina e delle sue miniere. Firenze: Tipografia Giuntina, 187 p., 31 tab., OCLC 11563375.

Sáez, R., Pascual, E., Toscano, M. \& Almodóvar, G. R. 1999. The Iberian type of volcano-sedimentary massive sulphide deposits. Mineralium Deposita 34:549-570

Sobek, A. A., Schuller, W. A., Freeman, J. R. \& Smith, R. M. 1978. Field and laboratory methods applicable to overburden and minesoils, EPA 600/278-054, 203 p.

Webb, J. 1958. Observations on the geology and origin of the San Domingos pyrite deposit. Portugal. Com. SGP, t. 42, 119-143. 